\title{
Optimization of Distribution Path considering Cost and Customer Satisfaction under New Retail Modes
}

\author{
Dengqing Wang $\mathbb{D}^{1,2}$ Yuting Yang, ${ }^{2}$ and Yanhu Wang ${ }^{3}$ \\ ${ }^{1}$ School of Economics and Management, Fuzhou University, Fuzhou 350108, China \\ ${ }^{2}$ School of Economics and Management, Fujian Chuanzheng Communications College, Fuzhou 350007, China \\ ${ }^{3}$ The Academy of Digital China (Fujian), Fuzhou University, Fuzhou 350108, China \\ Correspondence should be addressed to Dengqing Wang; 178477427@qq.com
}

Received 21 May 2021; Revised 24 July 2021; Accepted 17 August 2021; Published 7 September 2021

Academic Editor: Yuan Gao

Copyright (c) 2021 Dengqing Wang et al. This is an open access article distributed under the Creative Commons Attribution License, which permits unrestricted use, distribution, and reproduction in any medium, provided the original work is properly cited.

\begin{abstract}
One of the top issues in logistics management and related research is to establish an effective distribution system that is adaptive to new retail and capable of lowering the cost of logistics while enhancing consumer satisfaction. Aimed at reversing the weak points of current logistics distribution patterns, a dual-objective bipolar model with optimal logistics cost and consumer satisfaction by restraining distribution time and load is tested in this paper to figure out the proper nodes and vehicle routes. Data from general and front warehouses of PuPu mall, a Fuzhou-based online retail enterprise, are made into a case study. Moreover, the immune algorithm and genetic algorithm are adopted to achieve the model solution. It is found that the immune algorithm is more efficient than the genetic algorithm in searching solutions, thus having better adaptivity and effectiveness, and also that the type of distribution vehicle plays a significant role in determining the total distribution cost.
\end{abstract}

\section{Introduction}

In recent years, online retail (also known as new retail) has shown a rapid growth trend. In 2020, China's online retail sales reached 11760.1 billion yuan, an increase of $10.9 \%$ over the previous year. Among them, the online retail sales of physical goods reached 9759 billion yuan with an increase of $14.8 \%$, accounting for $24.9 \%$ of the total retail sales of social consumer goods. Logistics generation and order generation are the two basic links of online retail, and the evolution of the combination mode of the two constantly reshapes the format of e-commerce, resulting in the rapid updating and iteration of new retail modes. At present, the mainstream of the industry consists of three trends: integrating offline with online (such as https://www.Suning.com), integrating online with offline plus self-run logistics service (such as https:// www.JD.com), and online order plus front warehouse distribution and delivery (such as https://www.pupumall.com, Fuzhou). The distribution networks of these new retail logistics have the following common characteristics: they directly face the terminal customers, they have adjacent distribution outlets for customers and fast circulation, there are high requirements for customized and timely delivery, and there is low certainty of distribution quantity and frequency. A key problem of the new retail industry to solve as well as a research hotspot in related fields is to figure out how to adapt to the changes of new retail logistics distribution to reduce logistics costs and improve customer satisfaction, the core of which is to optimize the logistics path, including the optimization of logistics nodes and the paths from terminal nodes to distribution terminals (addresses of online shopping customers).

Scholars at home and abroad have taken large-scale online-to-offline $(\mathrm{O} 2 \mathrm{O})$ retail enterprises (such as Suning Appliance), their offline experience stores and distribution networks, and their terminal customers (mainly in cities where e-commerce is well developed) as research objects to reach various research targets $[1,2]$, such as minimizing total logistics cost, minimizing driving distance $[3,4]$, achieving greater customer satisfaction with the optimal distribution 
cost, or having the best goods circulation ability and logistics service level. The proposed models were solved by various algorithms to establish the optimal logistics distribution network nodes and paths [5-8]. Generally, the genetic algorithm [9-12] and immune algorithm [13-15] are widely applied to solve practical models. For example, Hou et al. [10] tried to develop a given refining schedule for the crude oil operations, which were transformed into an assignment problem of charging tanks and distillers. The problem was solved by a genetic algorithm. Wang et al. [11] constructed a multiperiod portfolio model by considering a conditional value-at-risk constraint for the multiperiod investment decisions. The model was solved by a variable neighborhood search-based hybrid genetic algorithm. Guo et al. [12] considered a multiobjective resource-constrained and sequence-dependent disassembly optimization problem with disassembly precedence constraints. This multiobjective optimization problem with a combinatorial nature was solved by a genetic algorithm efficiently.

However, some parts have still been left behind and need to be further tapped into the literature. First, there has not been pertinent and comprehensive research on the logistics distribution networks of regional new retail enterprises in China. Second, there is no comprehensive analysis of the influencing factors of customer satisfaction and minimizing the logistics cost of outlets has not been involved in the overall consideration. Third, there is no in-depth analysis of the impact of the influencing factors on customers' choice of front warehouses on logistics network operating costs.

In view of the limitations of the existing research results on new retail logistics terminal node layout and considering customer satisfaction and total logistics cost, this paper constructs a dual-objective bilevel programming model for site selection and layout optimization of new retail distribution centers and their front warehouses. An immune algorithm is adopted to seek solutions, aiming to provide technical support for solving new retail logistics problems.

\section{Problem Prototypes and Models}

2.1. Problem Description. Based on the logistics distribution network of https://www.pupumall.com, a regional new retail enterprise, this paper investigates the solutions to this kind of network by modeling, which involves optimizing distribution lines from the general warehouse to each front warehouse, selecting the sites of front warehouses, and covering the service area. The general warehouse is responsible for storage, sorting, transportation, distribution, distribution processing, order information processing, and so on, as well as delivering goods to the front warehouses within the region. The front warehouses are located where there is easy and quick access to customers and are responsible for temporary storage, transfer, sorting, order processing, and other functions, and it is required that goods be delivered within 30 minutes after receiving a customer's order. Light trucks are used for transportation from the general warehouse to the front warehouses, while electric vehicles are used from the front warehouses to the terminal customers. The total cost of logistics distribution includes both the transportation cost from the general warehouse to the front warehouses and the distribution cost from front warehouses to customers.

Customer satisfaction with the front warehouses is determined by factors such as distribution time, pickup distance, service price, service quality, and goods turnover ability [7]. As https://www.pupumall.com delivers goods directly to the door without the need for customers to pick up the goods themselves, customer satisfaction mainly depends on the distribution time, since the logistics distribution market is quite transparent and the prices are consistent, so they do not need to be considered. Therefore, customer satisfaction with the front warehouses is mainly influenced by three factors: distribution time, turnover capacity, and service quality.

2.2. Solution Model. To facilitate the search for a solution and increase the practical value of the model, the following assumptions are made for the logistics networks in this study:

(i) There is only one general warehouse, which is responsible for supplying goods to each front warehouse

(ii) The maximum processing capacity of an alternative front warehouse is the same as that of those in use, and the location is known

(iii) Electric vehicles are employed to transport goods from front warehouses to terminal customers and are capable of circular distribution

(iv) The maximum capacity of each vehicle is limited

(v) The demand for goods from each front warehouse is met, and only one vehicle services the goods

(vi) The length of each distribution path shall not exceed the maximum driving distance of the vehicle in service

(vi) The fixed cost of each front warehouse is different

(vii) The delivery time from a front warehouse to a customer is expected to be within 30 minutes

Moreover, the model sets, parameters, and decision variables used throughout the paper are as follows:

Sets:

$K$ is the set of vehicles used between the general warehouse and front warehouses

$N$ is the set of vehicles used between the general warehouse and front warehouses

$I$ is the collection of front warehouses

$J$ is the collection of locations of terminal customers

$N$ is the collection of terminal distribution of electric vehicles

Index:

$o$ is the index of general warehouse

$k$ is the index of vehicles of vehicle set $K$

$n$ is the index of vehicles of vehicle set $N$

$i$ is the index of front warehouses

$j$ is the index of terminal customers 
Parameters:

$c_{1}$ is the transportation rate from the general warehouse to a front warehouse (RMB $/(\mathrm{kg} \cdot \mathrm{km})$ )

$c_{2}$ is the transportation rate from a front warehouse to a terminal customer $(\mathrm{RMB} /(\mathrm{kg} \cdot \mathrm{km}))$

$k_{1}$ is the weight of the customer satisfaction on distribution time

$k_{2}$ is the weight of the customer satisfaction on turnover capacity

$k_{3}$ is the weight of the customer satisfaction on service quality

$d_{o i}$ is the distance from the general warehouse to a front warehouse, and $d_{i j}$ represents the distance from a front warehouse to a terminal customer

$q_{i}$ is the freight volume of each front warehouse $i$

$p_{j}$ is the demand quantity of customers $j$

$f_{i}$ is the fixed operating cost of each front warehouse $x_{\text {max }}$ is the service level of the front warehouse $\bar{t}_{i j}^{1}$ is the starting time point of the expected time window of customer $j$ on warehouse $i$

$\bar{t}_{i j}^{2}$ is the ending time point of the expected time window of customer $j$ on warehouse $i$

$\bar{t}_{i j}^{3}$ is the maximum acceptance time of customer $j$ on warehouse $i$

$L_{i j}$ is the distance between customer $j$ and warehouse $i$ $Q_{k}$ is the capacity of vehicle $k, k \in K$

$Q_{n}$ is the capacity of vehicle $n, n \in N$

$D_{k}$ is the driving distance capacity of vehicle $k, k \in K$

$D_{n}$ is the driving distance capacity of vehicle $n, n \in N$

Decision variables:

$x_{\text {oik }}$ binary variable, 1 indicates moving goods from the general warehouse to front warehouses $i$ by vehicle $k$

$x_{i j n}$ binary variable, 1 indicates moving goods from the front warehouse $i$ to terminal customer $j$ by electric vehicles $n$

$z_{i j}$ binary variable, 1 if customer $j$ is designated to warehouse $i$

$y_{i}$ binary variable, 1 indicates the front warehouse being selected

$s_{1 i}$ is the customer satisfaction of customer $i$ on distribution time

$s_{2}$ is the customer satisfaction of customer $i$ on turnover capacity

$s_{3 i}$ is the customer satisfaction of customer $i$ on service quality

$s t_{i j}$ is the customer satisfaction of customer $i$ on distribution time regarding $j$

$s x_{i}$ is the customer satisfaction of customer $i$ on turnover capacity

Based on the description of the problem and the variables, an optimization model of logistics outlets under the two-stage new retail model is established.
$\operatorname{Min} Y=Z+\frac{\lambda}{S}$

$$
Z=\sum_{i \in I} \sum_{k \in K} c_{1} q_{i} d_{o i} x_{o i k}+\sum_{i \in I} \sum_{j \in J} \sum_{k \in K} c_{1} q_{i} d_{o i} x_{o i k}+\sum_{i \in I} \sum_{k \in K} f_{i} y_{i}
$$

$$
\begin{aligned}
S & =\sum_{i \in I}\left(k_{1} \cdot s_{1 i}+k_{3} \cdot s_{3 i}\right)+k_{2} \cdot s_{2}, \\
s_{1 i} & =\sum_{j=1}^{m} \frac{s t_{i j}}{|J|}, \quad \forall i \in I, \\
s_{2} & =\sum_{i=1}^{n} \frac{s x_{i}}{|I|}, \\
s_{3 i} & =\sum_{i=1}^{n} \sum_{j=1}^{m} \frac{y_{i j}}{|J|}, \quad \forall i \in I,
\end{aligned}
$$

$s t_{i j}= \begin{cases}1, & \bar{t}_{i j}^{1} \leq t_{i j} \leq \bar{t}_{i j}^{2}, \\ 1-\frac{t_{i j}-\bar{t}_{i j}^{2}}{\bar{t}_{i j}^{3}-\bar{t}_{i j}^{2}}, & \bar{t}_{i j}^{2} \leq t_{i j} \leq \bar{t}_{i j}^{3}, 0, t_{i j}>\bar{t}_{i j}^{3},\end{cases}$

$s x_{i}= \begin{cases}1, & x_{i} \leq x_{\max }, \\ 1-\left(\frac{x_{i}-x_{\max }}{\alpha x_{\max }}\right)^{\beta}, & x_{i}>x_{\max }\end{cases}$

$\sum_{o \in r} \sum_{i \in I} x_{o i k}=1, \quad \forall k \in K$

$$
\sum_{i=1}^{n} q_{i} x_{o i k} \leq Q_{k}, \quad \forall k \in K,
$$

$\sum_{i=1}^{n} \sum_{j=1}^{m} p_{j} x_{i j n} \leq Q_{n}, \quad \forall n \in N$,

$$
\sum_{i=1}^{n} d_{o i} x_{o i k} \leq D_{k}, \quad \forall k \in K
$$

$\sum_{j=1}^{n} d_{i j} x_{i j n} \leq D_{n}, \quad \forall n \in N$,

$$
\begin{aligned}
\sum_{i \in I} \sum_{j \in J} x_{i j n} & =1, \\
z_{i j} & \leq y_{i}, \quad \forall i \in I, j \in J, \\
q_{i} & \geq \sum_{j \in J} p_{j} z_{i j}, \quad \forall i \in I,
\end{aligned}
$$




$$
\frac{L_{i j}}{v} \leq 0.5+\left(1-z_{i j}\right) M, \quad \forall i \in I, j \in J .
$$

The objective function of this paper features cost minimization and customer satisfaction maximization, thus taking the minimum value of $Z$ in formula (2) and the maximum value of $S$ in formula (3) with no direct weighting and obtaining the minimum value by calculating the reciprocal of satisfaction degree. Because the order of magnitude between the two functions is not uniform, the transformation factor $\lambda$ is introduced to transform the dualobjective model into a single-objective model, as shown in formula (1). To be specific, formula (2) shows the objective function of the total cost, which consists of the transportation cost from the general warehouse to the front warehouses, the distribution cost from the front warehouses to terminal customers, and the fixed operating cost of the front warehouses. Formula (3) represents the function of customer satisfaction, which is determined by three factors, namely, the distribution time $\left(s_{1 i}\right)$, goods turnover capacity $\left(s_{2}\right)$, and service quality $\left(s_{3 i}\right)$, which can be obtained by formulas (4)-(6). In formula (4), $s t_{i j}$ represents the membership degree of terminal customers $j$ to a front warehouse $i$ in terms of distribution time, which can be calculated using formula (7). Similarly, the customer satisfaction of customer on turnover capacity involved in formula (5) can be calculated by formula (8), in which $\alpha$ and $\beta$ in the function represent the customized coefficients for customers.

The above two objective functions have the nature of conflict. In other words, given a certain number of vehicles, if we reduce the transportation cost, distribution cost, and fixed operating cost, customer satisfaction will be improved. In this case, there is no conflict. However, the first objective function is a minimal one and the second objective function is a maximal one. Once one of them is optimized, the other objective would be impaired for certain.

Formula (9) presupposes that goods from a front warehouse can be delivered by only one vehicle. Formulas (10) and (11) guarantee that the goods loaded on the vehicle does not exceed its loading capacity. Similarly, formulas (12) and (13) presuppose that the length of the distribution line does not exceed the driving distance capacity. Formula (14) determines that each customer should be designated to one of the front warehouses. Formula (15) presumes that customers $j$ can be served by the front warehouse $i$ only when the front warehouse is selected. Formula (16) presupposes that the amount of goods in a front warehouse is not less than the amount demanded by its terminal customers. Formula (16) presets the load limit of the electric vehicle assigned to a front warehouse. Formula (17) presumes that the delivery time from a front warehouse to its customers shall not exceed 0.5 hours.

\section{Algorithm Solutions}

The immune algorithm is a new calculation method inspired by the antigens and antibodies of biological immune systems. It has the self-adaptive characteristic of obtaining the optimal solution of a multiobjective function by multiple mechanisms and thus can avoid being "premature" (converging to a local extreme value) to a large extent. In the immune algorithm, antigens correspond to problems to be solved and antibodies correspond to solutions. In the process of seeking a solution, the algorithm generates the optimal solution by simulating the biological immune system. The specific algorithm flow is as follows. First, input the antigen and expect the initial antibody to be generated and then calculate the affinity between antibodies; when the antibodies are varied to cause the metabolism of immune cells, new antibodies are generated by the selection, intersection, mutation operators, and immune operators. Under the influence mechanism of a set of judgment conditions, the antibody population of the immune algorithm is constantly adjusted, updated, and evolved, to search for the optimal antibody, that is, the optimal solution to the problem.

3.1. Antigen Input. The objective function and restrictive conditions of the problem are input as the antigen of the immune algorithm, and the solution to the problem is accordingly regarded as the antibody.

3.2. Initial Antibody Generation. In the algorithm, the immune system generates the initial antibody by searching memory cells, activating the memory function of the immune mechanism. After the memory mechanism obtains the solution (antibody) to a problem, it selects and retains a certain scale of high-quality individuals. In the process of antibody renewal, many excellent individuals are stored and the inferior ones are replaced in the memory bank.

3.3. Affinity Calculation. The affinity is a concept representing the relationship between an antigen and an antibody. The former is done with a fitness function $(F)$ to extract the target function $(Y)$. The latter suggests the similarity between antibodies, which is calculated with the following formula:

$$
S_{v, s}=\frac{k_{v, s}}{L}
$$

In this formula, $k_{v, s}$ refers to the same digits of two antibodies and $L$ refers to the length of the antibody.

Antibody concentration (i.e., the proportion of similar antibodies in the population) is calculated with the following formula:

$$
C_{v}=\frac{1}{R} \sum_{j \in R} S_{v, s}
$$

In this formula, $R$ is the total number of antibodies and $S_{v, s}=\left\{\begin{array}{ll}1, & S_{v, s}>T, \\ 0, & \text { otherwise }\end{array}\right.$, with $T$ serving as a preset threshold.

\subsection{Antibody Metabolism (Intersection and Variation).}

The metabolism of immune cells is caused by the intersection and variation of antibodies. In this paper, two randomly generated numbers are used as insertion points to 
exchange customer information, and the same elements in the new antibody are successively deleted while the missing customer numbers are filled back in to form two new legal antibodies. To let the filial generation inherit more genes of the parental generation, an evolutionary reversal operator mutation method is adopted to generate a new gene string. After comparing the fitness of the new antibody and the original antibody, the one with higher fitness enters the next generation.

3.5. Promotion and Inhibition of Antibody Generation. In the process of finding the optimal solution, when the antibody concentration exceeds a certain value, the solution process will converge too early to get a globally optimal solution. The problem of too many antibodies or too many similar antibodies can be solved by controlling antibody concentration to prevent population degeneration.

Formula (19) calculates the antibody concentration. If individuals with higher concentration are defined as $A_{1}, A_{2}, \ldots$, and $A_{t}$, the concentration probability of $t$ individuals can be calculated by the following formula:

$$
P_{d}=\frac{1}{N}\left(1-\frac{t}{N}\right) \text {. }
$$

When $1<t<N$, the concentration probability of the other $N-t$ individuals is

$$
P_{d}=\frac{1}{N}\left(1+\frac{t^{2}}{N^{2}-N \cdot t}\right) .
$$

The individual selection probability $(P)$ is made up of the fitness probability $\left(P_{f}\right)$ and concentration probability $\left(P_{d}\right)$ :

$$
\begin{aligned}
P_{f} & =\frac{1}{Y}, \\
P & =\delta \cdot P_{f}+(1-\delta) \cdot P_{d} .
\end{aligned}
$$

In the above formulas, $\delta$ is the affinity coefficient, $\delta>0$, and $P_{f}$ and $P_{d}<1$. Equation (23) suggests that the greater the individual fitness, the greater the selection probability, whereas the greater the individual concentration, the smaller the selection probability. Thus, not only can individuals with high fitness be retained, but also the diversity of individuals can be ensured, better avoiding premature convergence.

3.6. Group Renewal. Two antibodies can be randomly selected to undergo mutation according to the mutation probability set in the previous steps and then intersect with each other. Steps 3-5 are repeated until the termination condition of the algorithm is met.

3.7. The Judgment of Termination Conditions. When all parameters have met the termination conditions of the algorithm, the algorithm stops when the maximum number of iterations is reached and the algorithm ends when the best

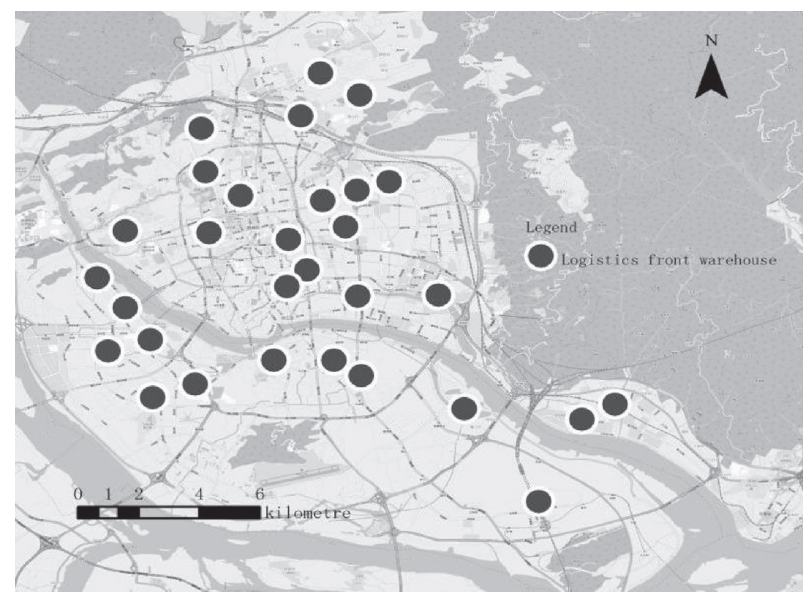

FIgURE 1: The layout of front warehouses.

individual is the optimal solution; otherwise, return to Step 3 and repeat the above cycle.

\section{Case Study}

4.1. Data and Model Parameters. A Fuzhou-based Internet retail enterprise, https://www.pupumall.com, was made a case study here. Its general warehouse is located in Zeyang village, Nantong, Minhou County, Fuzhou, and it has 30 front warehouses available. Each front warehouse is responsible for the distribution of goods to several communities. The layout of the front warehouses is shown in Figure 1, and the quantity demand of each warehouse is shown in Table 1. Customers in 298 communities are covered by the service offered by https://www.pupumall. com. The parameters involved in the model are shown in Table 2.

4.2. Model Solution Output. The parameters of the immune algorithm are as follows: population size $N=80$, crossover probability $P_{c}=0.8$, mutation probability is 0.2 , and iteration times are 100 . The algorithm runs 80 times before finding the final solution, and the minimum value of the total cost is 960,630 . Table 3 and Figure 2 compare the genetic algorithm and the immune algorithm, and the data suggest that each index of the immune algorithm is better than that of the genetic algorithm. The corresponding routes from the general warehouse to the front warehouses are shown in Figure 3, and the corresponding routes from front warehouses to customers are shown in Figure 4.

After 90 and 80 iterations, the genetic algorithm and immune algorithm reach the maximum fitness value of 0.762 . Meanwhile, the average time needed for the immune algorithm to converge to get the final solution is 97.8 seconds, 9.2 seconds faster than the genetic algorithm. It can be seen that the immune algorithm has better convergence and stability. Besides, compared with the genetic algorithm, the immune algorithm used in this paper can facilitate getting the final routes of vehicles in a shorter time. 
TABLE 1: Quantity demand of each front warehouse $(\mathrm{kg})$.

\begin{tabular}{lccccccccc}
\hline$q_{1}$ & $q_{2}$ & $q_{3}$ & $q_{4}$ & $q_{5}$ & $q_{6}$ & $q_{7}$ & $q_{8}$ & $q_{9}$ & $q_{10}$ \\
2200 & 1800 & 1100 & 1700 & 3000 & 2000 & 2300 & 1400 & 500 & 1600 \\
\hline$q_{11}$ & $q_{12}$ & $q_{13}$ & $q_{14}$ & $q_{15}$ & $q_{16}$ & $q_{17}$ & $q_{18}$ & $q_{19}$ \\
1900 & 400 & 1000 & 2400 & 600 & 3300 & 3800 & 2000 & 1500 & 2000 \\
\hline$q_{21}$ & $q_{22}$ & $q_{23}$ & $q_{24}$ & $q_{25}$ & $q_{26}$ & $q_{27}$ & $q_{28}$ & $q_{29}$ & $q_{30}$ \\
1300 & 1200 & 2100 & 2200 & 1900 & 1400 & 1200 & 1600 & 2700 & 1200 \\
\hline
\end{tabular}

TABle 2: Parameters involved in the model.

\begin{tabular}{lccccccc}
\hline$C_{1}$ & $C_{2}$ & $K_{1}$ & $K_{2}$ & $K_{3}$ & $\alpha$ & $\beta$ & \\
0.003 & 0.002 & 0.4 & 0.3 & 0.3 & 1 & 2 & 3000 \\
\hline$t_{1 i j}$ & $t_{2 i j}$ & $t_{3 i j}$ & $\lambda$ & $Q_{k}$ & $D$ & $L$ & $N_{k}$ \\
0 & 0.5 & 2 & 10000 & 4000 & 100 & 1.5 \\
\hline
\end{tabular}

TABle 3: Comparison between the genetic algorithm and immune algorithm.

\begin{tabular}{lccccc}
\hline Method & Total cost & Satisfaction value & Dual-objective & Iteration times & Max. fitness \\
\hline Genetic algorithm & 972,667 & 1.198 & 981,014 & 90 & 0.762 \\
Immune algorithm & 960,630 & 1.300 & 968,322 & 80 & 0.762 \\
\hline
\end{tabular}

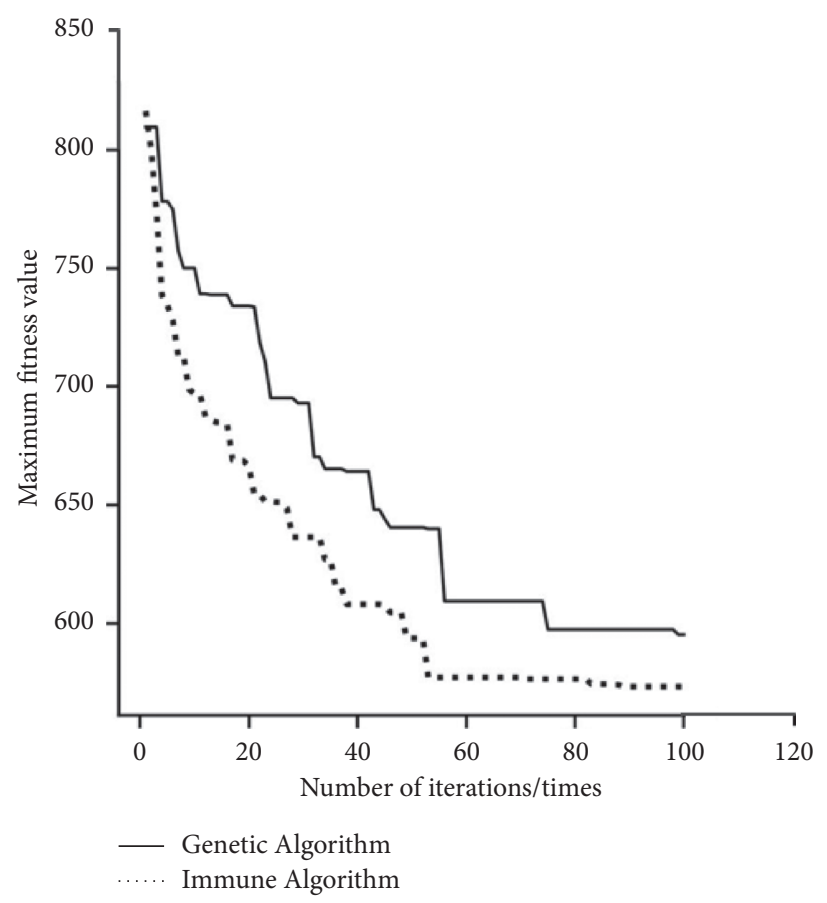

Figure 2: Fitness curve of immune and genetic algorithms.

We calculated the impact of two-vehicle models on the total cost of new retail logistics distribution. Table 4 shows that, with a 5 -ton vehicle load, the total cost is $1.08 \%$ which is less than that of a 4 -ton load, even with $20 \%$ fewer vehicles required, yet with better maximum fitness. It is therefore quite obvious that selecting vehicles with the same loading capacity as the demand capacity of the front warehouse will likely reduce the cost of logistics distribution. 


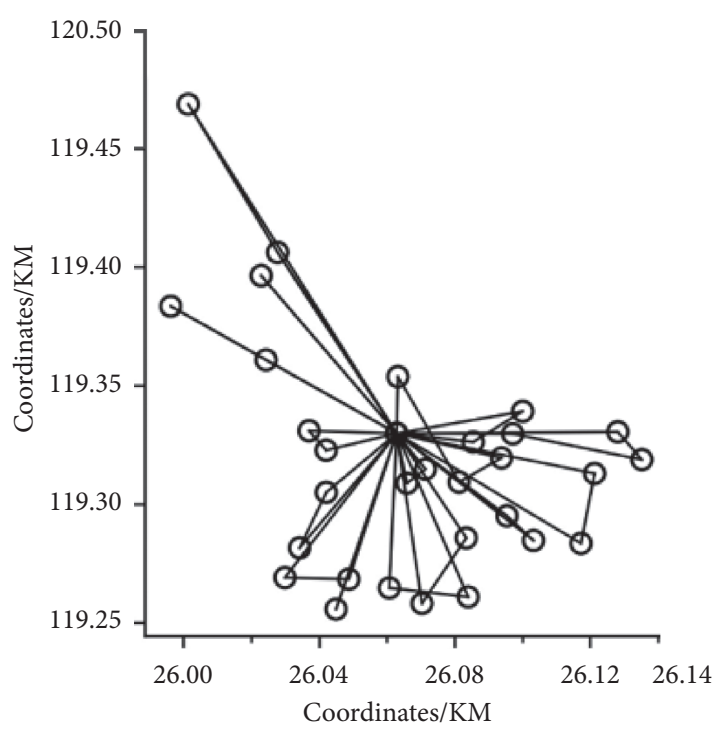

FIGURE 3: Returned routes from the general warehouse to the front warehouses.

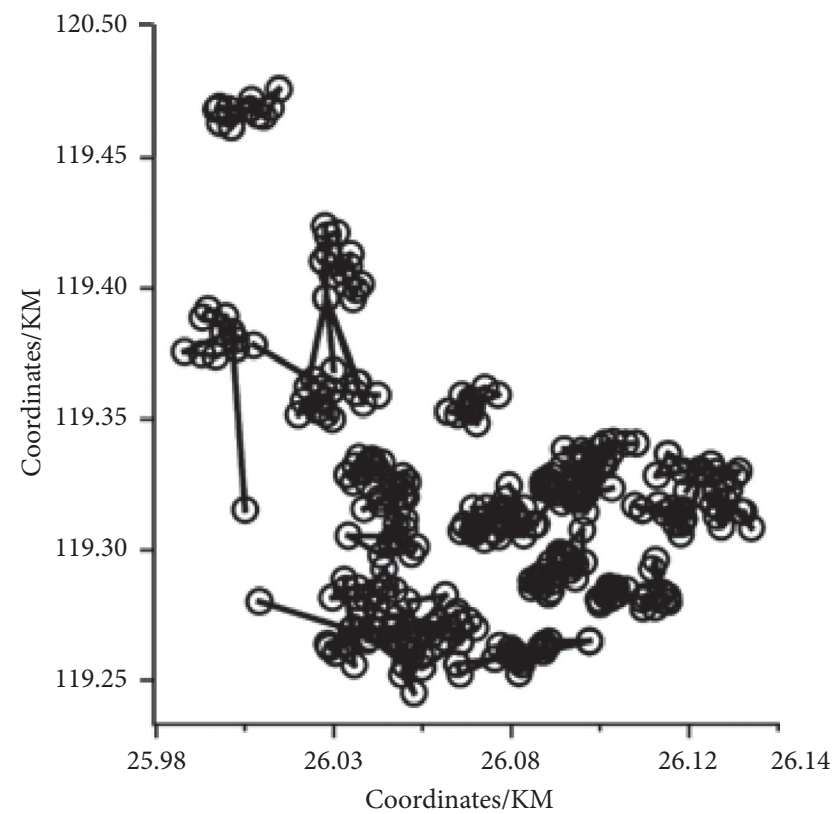

Figure 4: Returned routes from front warehouses to customers.

TABle 4: Comparison of types of vehicles used in new retail network distribution.

\begin{tabular}{lcccc}
\hline $\begin{array}{l}\text { Vehicle } \\
\text { load }\end{array}$ & $\begin{array}{c}\text { Total } \\
\text { cost }\end{array}$ & $\begin{array}{c}\text { Number of } \\
\text { iterations }\end{array}$ & $\begin{array}{c}\text { Number of } \\
\text { vehicles }\end{array}$ & $\begin{array}{c}\text { Maximum } \\
\text { fitness value }\end{array}$ \\
\hline 4 & 960,630 & 80 & 15 & 0.762 \\
5 & 950,219 & 92 & 12 & 0.763 \\
\hline
\end{tabular}

\section{Conclusions}

This paper focuses on optimization problems of logistics nodes and vehicle paths that need to be solved jointly for new retail enterprises (such as https://www.pupumall.com) using the operation mode of online order plus front warehouse distribution and delivery. A dual-objective optimization model is therefore established with the minimum logistics cost and maximum customer satisfaction. Data of logistics distribution from the general warehouse and front warehouses of https://www.pupumall.com were made into a case study, and the immune and general genetic algorithms were used to obtain model solutions. The conclusions are as follows.

First, the immune algorithm adopted to solve the optimization model established in this paper is capable of effectively preventing the degradation of the genetic algorithm in the later stage and is superior to the genetic algorithm in search speed, fitness, and optimization effect.

Second, with the restrictive conditions in this paper taken into consideration, the case study shows that the cost of the corresponding logistics distribution scheme suggested by the immune algorithm is lower than the corresponding index value of the genetic algorithm, and the former is more practical. Besides, the logistics distribution vehicle model has a significant impact on the total logistics cost. The vehicle model with the same loading capacity as the demand capacity of the designated front warehouse is more likely to reduce the distribution cost.

In this paper, it is presumed that the number of end nodes is limited and the road traffic is smooth when conducting the optimization. It will be the goal of the follow-up study to obtain a more practical solution by expanding the search scope to fully cover the service area and account for the situation of traffic congestion and restriction.

\section{Data Availability}

The data used to support the findings of this study are included within this article.

\section{Conflicts of Interest}

The authors declare that there are no potential conflicts of interest.

\section{Acknowledgments}

This paper was funded by the logistics research team of Chuanzheng Communications College (205165).

\section{References}

[1] Q. W. Zhao, J. P. Zhao, and Y. Lin, "A city logistics network optimization model for large chain retailers under online-offline channel integration," Chinese Journal of Management Science, vol. 25, pp. 159-167, 2017.

[2] Y. Hu, "The optimization of logistics distribution network nodes in retail enterprises under 020 mode," Business Economy Research, vol. 8, pp. 72-74, 2018.

[3] J. Berger and M. Barkaoui, "A new hybrid genetic algorithm for the capacitated vehicle routing problem," Journal of the Operational Research Society, vol. 54, no. 12, pp. 1254-1262, 2017.

[4] A. T. Mulloorakam and N. Mathew Nidhiry, "Combined objective optimization for vehicle routing using genetic 
algorithm," Materials Today: Proceedings, vol. 11, pp. 891-902, 2019.

[5] R. Cheng, "Research on distribution route optimization of B2C online supermarket based on data fusion algorithms under new retail mode," Basic and Clinical Pharmacology and Toxicology, vol. 125, no. 1, pp. 166-167, 2019.

[6] H. Pan, "Logistics distribution center location based on model optimization and immune optimization algorithm," Electronic Design Engineering, vol. 27, no. 10, pp. 78-81, 2019.

[7] C. Lu, J. Fang, and S. Fu, "A new equilibrium strategy of supply and demand for the supply chain of pig cycle," Mathematical Problems in Engineering, vol. 2020, Article ID 2093593, 13 pages, 2020.

[8] B. Wang, H. Zhang, J. Nie et al., "Multipopulation genetic algorithm based on GPU for solving TSP problem," Mathematical Problems in Engineering, vol. 2020, Article ID 1398595, 8 pages, 2020.

[9] T. Mareda, L. Gaudard, and F. Romerio, "A parametric genetic algorithm approach to assess complementary options of large scale windsolar coupling," IEEE/CAA Journal of Automatica Sinica, vol. 4, no. 2, pp. 260-272, 2017.

[10] Y. Hou, N. Wu, M. Zhou, and Z. Li, "Pareto-optimization for scheduling of crude oil operations in refinery via genetic algorithm," IEEE Transactions on Systems, Man, and Cybernetics: Systems, vol. 47, no. 3, pp. 517-530, 2017.

[11] J. Wang, M. Zhou, X. Guo, and L. Qi, "Multiperiod asset allocation considering dynamic loss aversion behavior of investors," IEEE Transactions on Computational Social Systems, vol. 6, no. 1, pp. 73-81, 2019.

[12] X. Guo, M. Zhou, S. Liu, and L. Qi, "Lexicographic multiobjective scatter search for the optimization of sequencedependent selective disassembly subject to multiresource constraints," IEEE Transactions on Cybernetics, vol. 50, no. 7, pp. 3307-3317, 2020.

[13] J. Qiao, F. Li, C. Yang, W. Li, and K. Gu, "A self-organizing RBF neural network based on distance concentration immune algorithm," IEEE/CAA Journal of Automatica Sinica, vol. 7, no. 1, pp. 276-291, 2020.

[14] L. Huang, M. Zhou, and K. Hao, "Non-dominated immuneendocrine short feedback algorithm for multi-robot maritime patrolling," IEEE Transactions on Intelligent Transportation Systems, vol. 21, no. 1, pp. 362-373, 2020.

[15] J. Chen, M. Zhou, and H. Zheng, "A novel radius adaptive based on center-optimized hybrid detector generation algorithm," IEEE/CAA Journal of Automatica Sinica, vol. 7, no. 6, pp. 1627-1637, 2018 . 